Rev Biomed 2000; 11:123-127.

\title{
La medicina legal o forense en Yucatán, México.
}

\author{
Historia de la Medicina
}

Arturo Erosa-Barbachano.

Ex-presidente de la Sociedad Yucateca de Historia y Filosofía de la Medicina.

Mérida, Yucatán, México.

\section{RESUMEN.}

En Mérida, Yucatán, México, desde su fundación en 1542 hasta 1580, el Dr. Juan del Rey fue médico del ejército y director del Hospital, por lo que intervino en algunos dictámenes médicolegales. Esta práctica continuó en los siglos siguientes.

En el siglo XVIII se creó en la ciudad de México, y por decreto real, una institución llamada Protomedicato, que tenía a su cargo algunas resoluciones del orden legal. No hubo en Yucatán este tipo de cuerpo colegiado; sin embargo, a principios del siglo XIX, el Dr. Cipriano Blanco fue nombrado Subdelegado del Protomedicato en Campeche.

En Mérida, a partir de 1804, las actuaciones oficiales quedaron a cargo del Dr. Alejo Dancourt hasta 1834 en que pasaron a responsabilidad del Dr. Ignacio Vado Lugo, en razón de su cargo de director de la Escuela de Medicina de Yucatán, hasta el año 1853 en que falleció. El 4 de octubre de 1869 se aprobó un nuevo plan de estudios para la Escuela de Medicina de Yucatán, estatuyendo la cátedra de Medicina Legal y Toxicología, que quedó a cargo del Dr. Juan Antonio Pérez Gálvez.

El 31 de marzo de 1882, el gobernador Octavio Rosado expidiera un decreto creando las plazas de dos médicos legistas y se nombró para ello a los doctores Juan Nicoli y Fernando Casares Martínez de Arredondo; el 16 de noviembre de 1883 fueron designados los Drs. Andrés Saénz de Santa María y García Rejón y José Jesús Castro López. Poco después, en 1889 en la Escuela de Medicina, el Dr. Sáenz ganó por oposición y en forma ad-vitam, la cátedra de Medicina Legal. En 1907, el Dr. Castro López renunció, por lo que fue nombrado en su lugar el Dr. Juan Wenceslao Miró y Pérez, quien estuvo en el servicio hasta 1913 en que lo sustituyó el Dr. José González Palma. En 1918 fue creado el Departamento MédicoLegal, designándose director general al Dr. Sáenz, quien desempeñó este cargo hasta el 31 de

Solicitud de sobretiros: Dr. Arturo Erosa-Barbachano. Calle 19-A No. 35 x 12, Col. Chuburna de Hidalgo, C.P. 97200, Mérida, Yucatán, México. Tel.: (9) 981-02-29 


\section{A Erosa-Barbachano.}

diciembre de 1924. Al jubilarse el Dr. Sáenz, ingresó al servicio el Dr. José Castro Fortuny. En 1942 fue nombrado el Dr. Avelino Ruiz Sansores. En 1945, se separó el Dr. Castro y fue suplido por el Dr. Oswaldo Riera Cardeña. En 1950 se designó al Dr. Gonzalo Marcín Cervera. El Dr. Riera renunció en 1951 y lo suplió el Dr. Víctor Manuel Nuñez Grintal, quien a su vez renunció en 1953, ocupando la plaza el Dr. Delio Angel Aguilar Vázquez. En enero de 1979 se designó para la jefatura al Dr. José Francisco Cambranis Puga en 1984 al Dr. Jorge Hadad Herrera, en 1986 al Dr. Juan M. Alvarez, y en 1990 el Dr. Fernando Olivares Alcocer. En 1995 asumió nuevamente la jefatura del Servicio Médico Forense el Dr. Hadad Herrera. En 1987 se fundó la Sociedad Yucateca de Medicina Forense, que agrupa a los profesionales de esta disciplina.

(Rev Biomed 2000; 11:123-127)

Palabras clave: Medicina forence, historia de la medicina.

\section{SUMMARY.}

Legal or forensic medicine in Yucatan, Mexico.

From the founding of the city of Merida, Yucatan, Mexico in 1542 to 1580, Dr Juan del Rey was the army doctor and the Director of the military hospital and therefore he intervened in some legalmedical reports. This practice continued during the following centuries.

In the eighteeth century an institution called "Protomedicato" was created in Mexico City by royal decree, this institution was responsible for some legal resolutions. There was no colegiate body of this kind in Yucatan, however Dr. Cipriano Blanco was named "Subdelegado del Protomedicato" in Campeche at the beginning of the nineteeth century.

From 1804, Dr. Alejo Dancourt was responsible for the official procedures in Merida until 1834 when Dr. Ignacio Vado Lugo assumed the responsability, being the Director of the School of Medicine of Yucatan, until he died in 1853. On October 4, 1869, a new plan of studies was approved by the School of Medicine of Yucatan, establishing the chair of Toxicology and Legal Medicine with Dr. Juan Antonio Pérez Galvez at its head.

On March 31, 1882, the Governer Octavio Rosado issued a decree creating positions for two forensic experts and named Dr. Juan Nicoli and Dr. Fernando Casares Martínez de Arredondo to occupy these positions; later on November 16 , 1883, Dr. Andres Saenz de Santa María y García Rejón and Dr. José de Jesús Castro López were designated for these positions. Then, in 1889 , there was a competitive examination for the Chair of Forensic Medicine in the School of Medicine which Dr. Saenz won. In 1907, Dr. Castro resigned and Dr. Juan Wenceslao Miro y Pérez was appointed to take his place and was on duty until 1913 when he was substituted by Dr. José González Palma. The Department of Forensic Medicine was created in 1918 and Dr. Saenz was designated General Director who occupied this post until December 31, 1924. On Dr. Saenz's retirement, Dr. José Castro Fortuny was appointed in his place. In 1942, Dr. González Palma died and Dr. Avelino Ruiz Sansores was appointed in his place. In 1945, Dr. Castro was substituted by Dr. Oswaldo Riera Cardeña. In 1950, Dr. Gonzalo Marcin Cervera was designated. Dr. Riera resigned in 1951 and was replaced by Dr. Delio Angel Aguilar Vasquez. In January 1979, Dr. José Francisco Cambranis Puga was designated for the leadership of the Department. In 1984, Dr. Jorge Hadad Herrera took up the leadership; in 1986 Dr. Juan M. Alvarez was named and he held the position until 1990 when he was taken over by Dr. Fernando Olivares Alcocer. In 1995, Dr. Hadad Herrera once again assumed the leadership of the foresic medical service. In 1987, the Yucatecan Society of Forensic Medicine, which grouped together all the foresic proffesionals, was founded. (Rev Biomed 2000; 11:123-127) 


\section{La medicina forense en Yucatán.}

Key words: Forensci medicine, history of medicine.

A partir de la época de la conquista, este aspecto de la medicina era aplicado a veces por los médicos militares, ya que eran los únicos médicos oficiales conque se contaba. En Mérida, Yucatán, México, desde su fundación en 1542 hasta 1580, el Dr. Juan del Rey fue médico del ejército y director del Hospital, por lo que intervino en algunos dictámenes médico-legales. Esta práctica continuó en los siglos siguientes.

En el siglo XVIII se creó en la ciudad de México, por ser capital del Virreinato de la Nueva España y por decreto real, una institución llamada Protomedicato, que tenía a su cargo algunas resoluciones del orden legal. No hubo en Yucatán este tipo de cuerpo colegiado; sin embargo, a principios del siglo XIX, el Dr. Cipriano Blanco fue nombrado Subdelegado del Protomedicato en Campeche.

En Mérida, a partir de 1804, las actuaciones oficiales quedaron a cargo del Dr. Alejo Dancourt hasta 1834 en que pasaron a responsabilidad del Dr. Ignacio Vado Lugo, en razón de su cargo de director de la Escuela de Medicina, hasta el año 1853 en que falleció.

Después del triunfo de las fuerzas republicanas sobre las imperialistas, el 4 de octubre de 1869 se aprobó un nuevo plan de estudios para la Escuela de Medicina de Yucatán, estatuyendo la cátedra de Medicina Legal y Toxicología, que quedó a cargo del Dr. Juan Antonio Pérez Gálvez. En octubre de 1873 se promulgó una nueva Ley de Instrucción Pública, que la prensa comentó en estos términos: "Considerada la Medicina en sus multiplicadas aplicaciones a las funciones del Gobierno, ha recibido el nombre de Medicina Política, que se ha dividido en dos ramas: la Higiene Pública y la Medicina Legal. Son ramas que no pueden separarse de su tronco común, la Medicina. Cuando el artículo 7 de la Ley Orgánica dispone que entre las asignaturas de la enseñanza en la Escuela de Medicina y Farmacia se cuenten la
Higiene y la Medicina Legal, tal disposición es sin duda muy certera. Mas cuando el artículo 6 de la misma Ley manda que en la enseñanza en la Escuela de Jurisprudencia comprenda la Medicina Legal, creemos que establece una obligación imposible." A pesar de esta opinión, se estableció.

Por otra parte, se acostumbraba que si los tribunales del Estado necesitaban un servicio pericial, se designaba a alguien para ello; en el caso de heridos, se nombraba a los médicos del Hospital. Así las cosas, en noviembre de 1878, el gobierno emitió un acuerdo señalando que los profesores de la Escuela de Medicina debían examinar a todos los heridos presentados a las autoridades; discutido en sesión, no se encontró justificación para ello y que podía poner en predicamento a la Escuela, con lo cual concordó el Dr. José Ma. Tappan que la dirigía y se formó una comisión para redactar las razones de la negativa, sugiriendo que eso podría ser función de un médico nombrado oficialmente para ello.

Posiblemente esto motivó que el 31 de marzo de 1882, el gobernador Octavio Rosado expidiera un decreto creando las plazas de dos médicos legistas y se nombré para ello a los doctores Juan Nicoli y Fernando Casares Martínez de Arredondo, pero al año siguiente ambos renunciaron, por lo que el 16 de noviembre de 1883 fueron designados los Drs. Andrés Saénz de Santa María y García Rejón y José Jesús Castro López.

Poco después, en 1889 en la Escuela de Medicina, el Dr. Sáenz ganó por oposición y en forma ad-vitam, la cátedra de Medicina Legal.

En 1907, uno de los legistas, el Dr. Castro López, renunció, por lo que fue nombrado en su lugar el Dr. Juan Wenceslao Miró y Pérez quien estuvo en el servicio hasta 1913 en que lo sustituyó el Dr. José González Palma. Durante los años que trabajó el Dr. Miró, publicó en las "Misceláneas" que editaba, muchos casos interesantes de asuntos médicos-legales.

En marzo de 1917. la Sociedad Médica Yucateca realizó una sesión en que su presidente, el Dr. Alberto Berrón Guerrero, presentó su trabajo 


\section{A Erosa-Barbachano.}

titulado "La Neurosífilis". A esta sesión fueron invitados los estudiantes de la cátedra de Medicina Legal de las Escuelas de Medicina y Jurisprudencia, llegándose a la conclusión de que los neurosifilíticos que han cometido un crimen deben ser recluídos para su curación y no puestos en libertad, con el argumento de ser irresponsables, sin tratamiento; los señores médicos legistas presentes manifestaron que ellos, en todos los informes que sobre el particular han rendido, han solicitado la reclusión y el tratamiento de estos enfermos pero que no saben, por ser éste asunto de resolución judicial, por qué han sido puestos en libertad.

En 1918 hubo una importante reorganización de las dependencias gubernamentales, siendo creado el Departamento Médico-legal, designándose director general al mismo Dr. Sáenz, quien desempeñó este cargo hasta el 31 de diciembre de 1924 en que se jubiló. Según sus cuidadosas estadísticas, de 1883 a 1924, o sea 41 años de trabajo del servicio de medicina legal, se emitieron 32,692 informes y se realizaron 3,533 autopsias, de las cuales 1,903 lo fueron en Mérida y 1,630 foráneas.

Al jubilarse el Dr. Sáenz, ingresó al servicio el Dr. José Castro Fortuny. En 1942 falleció el Dr. González Palma, por lo que fue nombrado en su lugar el Dr. Avelino Ruiz Sansores.

Poco después, en 1945, se separó el Dr. Castro y fue suplido por el Dr. Oswaldo Riera Cardeña. En 1950 se aumentó una plaza más y se designó al Dr. Gonzalo Marcín Cervera. El Dr. Riera renunció en 1951 y lo suplió el Dr. Víctor Manuel Nuñez Grintal quien a su vez renunció en 1953, ocupando la plaza el Dr. Delio Angel Aguilar Vázquez.

Esbozaremos ahora como eran las condiciones en que se trabajaba. Desde su fundación el servicio no tuvo oficina; cada médico despachaba en su propio consultorio o en su domicilio, según la hora, otras veces en la cárcel o en el hospital; las autopsias en Mérida, Yucatán se hacían en el hospital y en los pueblos se realizaban en los cementerios. En relación con este tema de las autopsias, parece que en épocas pasadas se utilizaba a estudiantes de medicina para efectuarlas, pero a final de los años treinta se designó ayudante oficial al bachiller Carlos Talavera León, que alguna vez se había inscrito en la Escuela de Medicina pero nunca aprobó el primer año; su pericia en las disecciones era notable y su sobrenombre popular, "Talín", se volvió legendario. Por falta de vehículo, los traslados al interior del Estado se hacían en tren, en camión de línea, a caballo o a pie. Otro gran problema era que tenían que desarrollar todo su poder de convencimiento para, vencer a veces la actitud negativa de los lugareños para el cumplimiento de la misión. En algunas ocasiones fueron protegidos de la equivocada indignación popular, en el calabozo del lugar, mientras llegaba la orden de la Procuraduría para que se diese garantías y la necesaria escolta para abandonar el lugar.

En 1968 se jubiló el Dr. Ruiz Sansores y ascendió a la jefatura del Servicio Médico Forense el Dr. Aguilar Vázquez, quien hizo una reestructuración y modernización del Servicio: se logró la dotación de un vehículo; se le proporcionó local propio en el edificio de la Procuraduría; se modernizó el mobiliario y equipo; se logró que hubiese un escribiente y se organizaron guardias para poder laborar las 24 horas.

Esta oficina de medicina legal dependía del Departamento de Identificación y Servicios Periciales, jefaturado por Ernesto Abreu Gómez, quien apoyó también que entraran a laborar varios médicos más.

En 1974, la Procuraduría de Justicia creó un Instituto de Capacitación para el personal, por lo que los médicos del servicio forense impartieron un curso somero de medicina legal a los agentes de la Policía Judicial.

Por causa de haber sufrido un infarto cardíaco, en enero de 1979 se jubiló el Dr. Aguilar Vázquez por lo que se designó para la jefatura al Dr. José Francisco Cambranis Puga. Al año siguiente, 1980, se dió al servicio la categoría de Departamento de Medicina Legal.

\section{Revista Biomédica}




\section{La medicina forense en Yucatán.}

En 1984 el Dr. Jorge Hadad Herrera ocupó la jefatura; ese año comenzó a funcionar la sala de necropsias en el cementerio de Xoclán; en 1986 solicitó licencia para realizar estudios de especialización en la capital de la República por lo que se nombró al Dr. Juan M. Alvarez en su lugar, ocupando el cargo hasta 1990 en que fue sustituido por el Dr. Fernando Olivares Alcocer. Durante su gestión, en 1993, se dotó al servicio de 2 ambulancias nuevas y se aumentó el número de médicos y personal auxiliar, ya que la demanda de servicios se había incrementado, llegando. a un promedio diario de 2 autopsias y 50 informes diagnósticos.

En 1995 asumió nuevamente la jefatura del servicio médico forense el Dr. Hadad Herrera. Actualmente la organización cuenta en el estado de Yucatán con una oficina central en Mérida y delegaciones en Valladolid, Tizimín, Ticul, Tekax, Progreso y Maxcanú, estando en organización la de Izamal; en total laboran 55 personas, de las que 26 son médicos con las especialidades siguientes: un psiquiatra, un radiólogo, un internista, un cirujano general, dos anatomo-patólogos forenses, tres médicos generales, seis doctoras especialistas en delitos sexuales y otros con adiestramiento en medicina legal.

En el laboratorio trabajan 8 químicos que realizan los exámenes toxicológicos, detección de drogas y otros, y se efectúan también los exámenes citológicos y de patología forense. La sala de autopsias de Xoclán cuenta con el necesario instrumental y equipo, incluyendo rayos $\mathrm{X}$, así como la labor auxiliar de 6 estudiantes de medicina. Se dispone de 5 ambulancias.

Se trabaja en 3 turnos. Durante 1999 se realizaron, en todo el estado de Yucatán, 870 autopsias y se rindieron más de 100,000 dictámenes y peritajes.

Por otra parte, en 1987 se fundó la Sociedad Yucateca de Medicina Forense, que agrupa a los profesionales de esta disciplina. El Dr. Hadad preside actualmente la Sociedad Yucateca de Medicina forense.

\section{BIBLIOGRAFIA.}

Aguilar V. Delio. 50 años de ejercicio profesional. Mérida: Revista del Colegio de Médicos de Yucatán 1993; 1:15-6.

Avila E. Alvaro. Historia de la Medicina en Yucatán. Mérida: Asociación Médica Mexicana; 1926.

Erosa B. Arturo. Historia de la Escuela de Medicina de Mérida. Mérida: Universidad Autónoma de Yucatán; 1998.

Erosa B. Arturo. Medicina Legal o Forense. Enciclopedia Yucatán en el Tiempo (en prensa). Mérida: Inversiones Care, S.A. de C.V.; 2000. 